\title{
Planck's Oscillators at Low Temperatures and Haken's Perturbation Approach to the Quantum Oscillators Reconsidered
}

\author{
Stanisław Olszewski \\ Institute of Physical Chemistry, Polish Academy of Sciences, Kasprzaka 44/52, Warsaw, Poland \\ Email: olsz@ichf.edu.pl
}

How to cite this paper: Olszewski, S. (2021) Planck's Oscillators at Low Temperatures and Haken's Perturbation Approach to the Quantum Oscillators Reconsidered. Journal of Modern Physics, 12, 1721-1728. https://doi.org/10.4236/jmp.2021.1212100

Received: September 21, 2021

Accepted: October 25, 2021

Published: October 28, 2021

Copyright $\odot 2021$ by author(s) and Scientific Research Publishing Inc. This work is licensed under the Creative Commons Attribution International License (CC BY 4.0).

http://creativecommons.org/licenses/by/4.0/

\section{(c) (i) Open Access}

\begin{abstract}
In the first step the extremal values of the vibrational specific heat and entropy represented by the Planck oscillators at the low temperatures could be calculated. The positions of the extrema are defined by the dimensionless ratios between the quanta of the vibrational energy and products of the actual temperature multiplied by the Boltzmann constant. It became evident that position of a local maximum obtained for the Planck's average energy of a vibration mode and position of a local maximum of entropy are the same. In the next step the Haken's time-dependent perturbation approach to the pair of quantum non-degenerate Schrödinger eigenstates of energy is re-examined. An averaging process done on the time variable leads to a very simple formula for the coefficients entering the perturbation terms.
\end{abstract}

\section{Keywords}

Planck's Quantum Oscillators at Low Temperatures, Calculation of the Extremal Specific Heat of the Oscillator Energy and Extremal Entropy, Simplified Haken's Time-Dependent Approach to the Perturbation Energy of a Non-Degenerate Quantum State

\section{Introduction}

Planck has developed-a time ago-a quantum approach to the oscillator ensembles for which both the vibrational energy and entropy are considered [1]. In the thermal equilibrium these parameters are given respectively by the formula

$$
E=N \varepsilon_{0}+N \frac{h v}{\mathrm{e}^{h v / k T}-1}
$$

in the energy case, and by 


$$
S=\frac{1}{T} \frac{N h v}{\mathrm{e}^{h v / k T}-1}-N k \log \left(1-\mathrm{e}^{-\frac{h v}{k T}}\right)
$$

in the entropy case. $N$ is the number of the component particles oscillating in the system:

$$
N_{0}+N_{1}+N_{2}+\cdots=N
$$

and

$$
N_{0} \varepsilon_{0}+N_{1} \varepsilon_{1}+N_{2} \varepsilon_{2}+\cdots=E
$$

is the energy $E$ given by $N$. This $E$ is composed by the groups of oscillating particles entering (3), symbols $\varepsilon_{i}$ denote the energy contributed by a single particle belonging to the set $N_{i}$.

The parameter $T$ is the absolute temperature, $v$-the symbol of frequency of the particle oscillation, $h$ and $k$ are the Planck and Boltzmann constants, respectively. We note that any oscillator has the energy

$$
\varepsilon_{n}=\varepsilon_{0}+n h v
$$

where $\varepsilon_{0}$ is a common energy component in the system.

Beyond of the general formulae for $E$ and $S$ presented in (1) and (2), Planck examined also a limiting situation when $T$ becomes very high. In this case we obtain

$$
\mathrm{e}^{h v / k T} \cong 1+\frac{h v}{k T}
$$

In effect of (6):

$$
\mathrm{e}^{h v / k T}-1 \cong \frac{h v}{k T}
$$

and by neglecting the term having $\varepsilon_{0}$-we obtain for energy

$$
E \cong N h v \frac{k T}{h v}=N k T \text {. }
$$

A substitution similar to (7) done in the case of $S$ in (2) gives (see [1]):

$$
S=N k \log \left(\frac{e k T}{h v}\right) .
$$

This is an approximate formula in which a small term having

$$
T \gg 0
$$

in the denominator in (7) has been neglected.

The low temperatures have not been much examined in [1]. In this case we have for the oscillatory energy

$$
E=N \varepsilon_{0}+N h v \mathrm{e}^{-h v / k T}
$$

because the first term entering the denominator in the formula (1) highly predominates over the absolute value of the second term of the denominator equal to 1 .

The idea of the present paper is to examine the case of very small $T$ more ac- 
curately than before. The examination of the energy behaviour is based mainly on the formula (11), a suitable basis of the examination of entropy is also taken from [1]; see Section 2.

The problem of the Planck oscillators for energy and entropy could be connected with the Haken's time-dependent perturbation calculation of a single nondegenerate quantum state. The solutions are shown to become similar to those of an oscillator-like equation having one term fully independent of time. Since an averaging process makes the time-dependent terms equal to zero, the coefficients entering the final solution approach the terms characteristic for the harmonic oscillator.

\section{Properties of Planck's $E$ and $S$ Characteristic for Small Temperature $T$}

Very small $T$ give for energy the formula (11), the same $T$ assumed for the entropy $S$ in (2) give (see [1]):

$$
S=\frac{N h v}{T} \mathrm{e}^{-h v / k T} .
$$

On the basis of $E$ in (11) we examine also its derivative with respect to $T$ representing the specific heat

$$
\frac{\mathrm{d} E}{\mathrm{~d} T}=N h v \frac{\mathrm{d}}{\mathrm{d} T}\left(\mathrm{e}^{-h v / k T}\right)=N h v \frac{\mathrm{de}^{-x}}{\mathrm{~d} x} \frac{\mathrm{d} x}{\mathrm{~d} T} .
$$

Here we put

$$
x=\frac{h v}{k T},
$$

so

$$
\frac{\mathrm{d} x}{\mathrm{~d} T}=-\frac{h v}{k T^{2}} .
$$

In effect the term (13) for the specific heat becomes:

$$
\frac{\mathrm{d} E}{\mathrm{~d} T}=N h v \frac{\mathrm{d}}{\mathrm{d} T}\left(\mathrm{e}^{-h v / k T}\right)=N h v(-1) \mathrm{e}^{-x}\left(-\frac{h v}{k T^{2}}\right)=N k x^{2} \mathrm{e}^{-x}=N k \frac{x^{2}}{\mathrm{e}^{x}} .
$$

In order to examine the external properties of (16) we calculate

$$
\frac{\mathrm{d}}{\mathrm{d} x}\left(\frac{x^{2}}{\mathrm{e}^{x}}\right)=\frac{\mathrm{d}}{\mathrm{d} x}\left(x^{2} \mathrm{e}^{-x}\right)=2 x \mathrm{e}^{-x}-x^{2} \mathrm{e}^{-x}=0
$$

which gives

$$
2 x=x^{2}
$$

or

$$
x=2 .
$$

The second derivative of the function examined in (17) gives

$$
\left.\frac{\mathrm{d}^{2}}{\mathrm{~d} x^{2}}\left(x^{2} \mathrm{e}^{-x}\right)\right|_{x=2}=\left.\mathrm{e}^{-x}\left(2-4 x+x^{2}\right)\right|_{x=2}=\mathrm{e}^{-2}(-2)<0,
$$


so the result in (20) indicates a local maximum of the function $\mathrm{d} E / \mathrm{d} T$ considered in (16):

$$
\left(\frac{\mathrm{d} E}{\mathrm{~d} T}\right)_{\max }=N k \frac{4}{\mathrm{e}^{2}} .
$$

A similar behaviour can be observed for the entropy at low $T$. This case of $S$ is represented by the formula given in [1] repeated in (12):

$$
S=N k x \mathrm{e}^{-x} .
$$

An examination of the derivative of $S$ leads to the result

$$
\frac{\mathrm{d} S}{\mathrm{~d} x}=N k \frac{\mathrm{d}}{\mathrm{d} x}\left(x \mathrm{e}^{-x}\right)=N k\left(\mathrm{e}^{-x}-x \mathrm{e}^{-x}\right)=N k \mathrm{e}^{-x}(1-x)
$$

from which we obtain the extremum at

$$
x=1 .
$$

The second derivative of $S$ in (22) gives

$$
\frac{\mathrm{d}^{2} S}{\mathrm{~d} x^{2}}=N k\left[-\mathrm{e}^{-x}-\mathrm{e}^{-x}-x\left(-\mathrm{e}^{-x}\right)\right]=N k(x-2) \mathrm{e}^{-x}
$$

which calculated at $x=1$ becomes:

$$
\left.\frac{\mathrm{d}^{2} S}{\mathrm{~d} x^{2}}\right|_{x=1}=-\frac{N k}{\mathrm{e}}<0 .
$$

The negative result in (26) indicates a maximum value of $S$ in (24). Evidently this extremal value of $S$ becomes:

$$
S_{\max }=\left.N k x \mathrm{e}^{-x}\right|_{x=1}=\frac{N k}{\mathrm{e}} .
$$

\section{Average Energy for the Planck's Vibration Mode and Its Properties}

This energy is presented in [2]:

$$
E_{a v}=\frac{h v}{\mathrm{e}^{h v / k T}-1}=\frac{h v}{\mathrm{e}^{x}-1} .
$$

The variable $x$ entering (28) [see (14)] taken at small $T$ can make $x$ much larger than 1. Evidently in this case we have

$$
\mathrm{e}^{x}=\mathrm{e}^{h v / k T} \gg 1
$$

and the term 1 entering the denominator in (28) can be neglected. Because of (14) we have approximately

$$
E_{a v}=f(x) \cong \frac{x}{\mathrm{e}^{x}} k T .
$$

This gives the energy derivative calculated with respect to $x$ equal to

$$
f^{\prime}(x)=\left(\frac{1}{\mathrm{e}^{x}}-\frac{x}{\mathrm{e}^{x}}\right) k T=(1-x) \frac{k T}{\mathrm{e}^{x}} .
$$

The requirement that $f^{\prime}(x)$ should be equal to zero gives 


$$
x=1
$$

which is the result much similar to that obtained in (24). The second derivative of $E_{a v}$ in (30) with respect to $x$ gives:

$$
f^{\prime \prime}(x)=k T\left(-2 \mathrm{e}^{-x}+x \mathrm{e}^{-x}\right)<0
$$

for $x=1$ indicating a maximum of (30) at that $x$.

\section{Similarities in Behaviour of the Derivatives of $E$ and $S$ Calculated with Respect to the Frequency $v$}

By taking first the derivative of the energy at low $T$ with respect to $v$ we obtain from (11):

$$
\begin{aligned}
\frac{\mathrm{d} E}{\mathrm{~d} v} & =\frac{\mathrm{d}}{\mathrm{d} v}\left(N h v \mathrm{e}^{-h v / k T}\right)=N k T \frac{\mathrm{d}}{\mathrm{d} x}\left(x \mathrm{e}^{-x}\right) \frac{\mathrm{d} x}{\mathrm{~d} v}=N k T \mathrm{e}^{-x}(1-x) \frac{\mathrm{d} x}{\mathrm{~d} v} \\
& =N h \mathrm{e}^{-x}(1-x)=N h \mathrm{e}^{-h v / k T}\left(1-\frac{h v}{h T}\right) .
\end{aligned}
$$

A much similar result can be calculated from the derivative of $S$ taken at low $T$ [see (12)]:

$$
\begin{aligned}
\frac{\mathrm{d} S}{\mathrm{~d} v} & =\frac{\mathrm{d} S}{\mathrm{~d} x} \frac{\mathrm{d} x}{\mathrm{~d} v}=\left[N k \mathrm{e}^{-x}+N k x(-1) \mathrm{e}^{-x}\right] \frac{\mathrm{d} x}{\mathrm{~d} v} \\
& =N k \mathrm{e}^{-x}(1-x) \frac{h}{k T}=\frac{N h}{T} \mathrm{e}^{-h v / k T}\left(1-\frac{h v}{k T}\right) .
\end{aligned}
$$

Both derivatives in (33) and (34) vanish at

$$
h v / k T=1 \text {. }
$$

The second derivatives of $E$ and $S$ calculated with respect to $v$ at $x=1$ give negative values which indicate positions of the maxima of $E$ and $S$ at the variable $x=1$ :

$$
\begin{aligned}
& E_{\max }=\frac{N h v}{\mathrm{e}}, \\
& S_{\text {max }}=\frac{N h v}{T \mathrm{e}},
\end{aligned}
$$

valid at low $T$.

\section{Oscillator Properties Representing the Haken's Time-Dependent Perturbation Approach to the Schrödinger's Quantum State}

This approach is rather special because it refers us directly to the time variable which, in general, is rather avoided by the quantum physicists.

Let us assume that only two separate quantum levels, say 1 and 2, of the unperturbed Hamilton eigenequation

$$
\hat{H}_{0} \varphi_{n}=W_{n} \varphi_{n}
$$

are for us of interest [3]. This implies that the solution of the time-dependent Schrödinger equation 


$$
i \hbar \frac{\mathrm{d} \psi}{\mathrm{d} t}=\hat{H} \psi
$$

where

$$
\hat{H}=\hat{H}_{0}+\hat{H}_{p}
$$

and $\hat{H}_{p}$ is the perturbation potential, is constructed with the aid of the combination of $\varphi_{1}$ and $\varphi_{2}$ entering (37):

$$
\psi=c_{1}(t) \varphi_{1}+c_{2}(t) \varphi_{2} .
$$

The $c_{1}(t)$ and $c_{2}(t)$ should be found. The equations defining the coefficients are:

$$
\begin{aligned}
& i \hbar \frac{\mathrm{d} c_{1}}{\mathrm{~d} t}=c_{1} W_{1}+c_{1} H_{11}^{p}+c_{2} H_{12}^{p}, \\
& i \hbar \frac{\mathrm{d} c_{2}}{\mathrm{~d} t}=c_{2} W_{2}+c_{2} H_{22}^{p}+c_{1} H_{21}^{p} .
\end{aligned}
$$

If we assume that the diagonal matrix elements of $\hat{H}_{p}$ vanish, i.e.

$$
H_{11}^{p}=H_{22}^{p}=0,
$$

a substitution can be done [3]:

$$
\begin{aligned}
& c_{1}(t)=d_{1} \exp \left(-i W_{1} t / \hbar\right), \\
& c_{2}(t)=d_{2} \exp \left(-i W_{2} t / \hbar\right) .
\end{aligned}
$$

This leads to the pair of equations for $d_{1}$ and $d_{2}$ equal respectively to [3]

$$
\begin{gathered}
i \hbar \frac{\mathrm{d}}{\mathrm{d} t} d_{1}=d_{2} H_{12}^{p} \exp (-i \tilde{\omega} t), \\
i \hbar \frac{\mathrm{d}}{\mathrm{d} t} d_{2}=d_{1} H_{21}^{p} \exp (i \tilde{\omega} t)
\end{gathered}
$$

where

$$
\tilde{\omega}=\omega_{21}=\left(W_{2}-W_{1}\right) \frac{1}{\hbar} .
$$

Our idea is to calculate the second derivatives of $d_{1}$ and $d_{2}$ entering (46) and (47) with respect to $t$ each multiplied by $i \hbar$ :

$$
\begin{aligned}
-\hbar^{2} \frac{\mathrm{d}^{2}}{\mathrm{~d} t^{2}} d_{1} & =i \hbar \frac{\mathrm{d}}{\mathrm{d} t}\left(d_{2}\right) H_{12}^{p} \exp (-i \tilde{\omega} t)+i \hbar d_{2} H_{12}^{p}(-i \tilde{\omega}) \exp (-i \tilde{\omega} t) \\
& =d_{1} H_{21}^{p} \exp (i \tilde{\omega} t) H_{12}^{p} \exp (-i \tilde{\omega} t)+d_{2} \hbar H_{12}^{p} \tilde{\omega} \exp (-i \tilde{\omega} t) \\
& =d_{1} H_{21}^{p} H_{12}^{p}+\hbar d_{2} H_{12}^{p} \tilde{\omega} \exp (-i \tilde{\omega} t)
\end{aligned}
$$

and

$$
\begin{aligned}
-\hbar^{2} \frac{\mathrm{d}^{2}}{\mathrm{~d} t^{2}} d_{2} & =i \hbar \frac{\mathrm{d}}{\mathrm{d} t}\left(d_{1}\right) H_{21}^{p} \exp (i \tilde{\omega} t)+i \hbar d_{1} H_{21}^{p} i \tilde{\omega} \exp (i \tilde{\omega} t) \\
& =d_{2} H_{12}^{p} \exp (-i \tilde{\omega} t) H_{21}^{p} \exp (i \tilde{\omega} t)-d_{1} \hbar H_{21}^{p} \tilde{\omega} \exp (i \tilde{\omega} t) \\
& =d_{2} H_{12}^{p} H_{21}^{p}-\hbar d_{1} H_{21}^{p} \tilde{\omega} \exp (i \tilde{\omega} t) .
\end{aligned}
$$

The results indicate that a part of the second derivative of both $d_{1}$ and $d_{2}$ is 
fully independent of $t$, being a multiple of $d_{1}$ and $d_{2}$ respectively. On the other side, the next part of each second derivative is very rapidly oscillating with $t$, because usually we have

$$
\tilde{\omega} \gg 1 ;
$$

see (48). This means that the average obtained for the time dependent part on the right of (49) and (50) is a very small number tending to zero. In effect we obtain the equations:

$$
-\hbar^{2} \frac{\mathrm{d}^{2}}{\mathrm{~d} t^{2}}\left(d_{1}\right) \cong d_{1} H_{21}^{p} H_{12}^{p}
$$

and

$$
-\hbar^{2} \frac{\mathrm{d}^{2}}{\mathrm{~d} t^{2}}\left(d_{2}\right) \cong d_{2} H_{12}^{p} H_{21}^{p}
$$

Because the coefficients on the right of (51) and (52) are the same, this imples that $d_{1}$ and $d_{2}$ can be represented respectively by the same function of time, say

$$
d_{1} \cong d_{2}=\cos v t \text {. }
$$

Since we have

$$
\frac{\mathrm{d}^{2}}{\mathrm{~d} t^{2}}\left(d_{1}\right) \cong \frac{\mathrm{d}^{2}}{\mathrm{~d} t^{2}}\left(d_{2}\right)=-v^{2} \cos (v t)
$$

the frequency square $v^{2}$ in (54) becomes

$$
v^{2}=\frac{H_{12}^{p} H_{21}^{p}}{\hbar^{2}} \sim \sec ^{-2}
$$

if we note that $H_{12}^{p} \sim H_{21}^{p} \sim \operatorname{erg}$ and $\hbar \sim$ erg $\cdot$ sec

We expect $v$ to be a large frequency because of a very small size of $\hbar$.

\section{Summary}

In the first step of the paper the extremal values of the specific heat of the Planck oscillator and the oscillator entropy are calculated. The results are attained by considering the well-established Planck's expressions for the oscillator energy and entropy in [1] taken for the limit of the low temperature $T$. As a variable $x$ suitable to the extrema calculations the dimensionless energy ratio (14) has been chosen.

It is found that the specific heat as well as entropy of the oscillators attains their maximal values given by the formulae (21)-(27), respectively, at $x=2$ and $x=1$. Both results, being proportional to the Boltzmann constant $k$, are small for $N=1$ but independent of $T$.

The limits of $\mathrm{d} E / \mathrm{d} T$ and $S$ obtained at very small $T$ become equal to zero for both specific heat and entropy:

$$
\lim _{T \rightarrow 0} \frac{\mathrm{d} E}{\mathrm{~d} T}=\lim _{x \rightarrow \infty} N k \frac{x^{2}}{\mathrm{e}^{x}}=0,
$$




$$
\lim _{T \rightarrow 0} S=\lim _{x \rightarrow \infty} N k \frac{x}{\mathrm{e}^{x}}=0 .
$$

In the next step the Haken's time-dependent perturbation method is discussed [3]. It is shown that the time-dependent perturbation coefficients representing this method can be obtained very easily on the basis of the harmonic oscillations having the frequency defined by the non-diagonal matrix element of the perturbation potential and the Planck constant $h$.

It should be noted that more recently the classical and quantum behaviour of the oscillations was examined with the aid of the linear canonical transformations in [4].

\section{Conflicts of Interest}

The author declares no conflicts of interest regarding the publication of this paper.

\section{References}

[1] Planck, M. (1932) Einführung in die Theorie der Wärme. 2nd Edition, S. Hirzel, Leipzig.

[2] Jauncey, G.E.M. (1948) Modern Physics. 3rd Edition, Van Nostrand, New York.

[3] Haken, H. (1986) Light Waves, Photons, Atom. Elsevier, Amsterdam.

[4] Ogura, A. (2016) Journal of Modern Physics, 7, 2205-2218. https://doi.org/10.4236/jmp.2016.715191 